\title{
Metabolomic Analysis of Liver from Dietary Biotin Deficient Mice
}

\author{
Toshinobu KuroISHI and Shunji SugaWARA \\ Division of Oral Immunology, Department of Oral Biology, Tohoku University Graduate \\ School of Dentistry, Sendai 980-8575, Japan
}

(Received April 29, 2019)

\begin{abstract}
Summary Biotin is a water-soluble B-complex vitamin that functions as a cofactor of five carboxylases. Because biotin-dependent carboxylases catalyze indispensable cellular metabolic functions, biotin deficiency is considered to be involved in various pathological conditions. Moreover, biotin supplementation shows pharmacological effects in vivo. However, the precise mechanisms by which biotin deficiency induces pathological conditions remain unclear. Although abnormal metabolites are used as indicators for biotin deficiency, few comprehensive analyses of total metabolites have been reported. In this study, we analyzed the metabolomic profiles of liver extracts prepared from biotin-sufficient (BS) and -deficient (BD) mice. Thirteen of 126 metabolites showed significantly different concentrations between liver extracts from BD and BS mice. The concentrations of 5 essential amino acids, Met, Val, Thr, Ile, and Leu, and 2 conditionally essential amino acids, Cys and Tyr were significantly lower in BD mice than in BS mice. Among these, the concentrations of sulfurcontaining amino acids, Cys and Met, were more than 1.5-fold lower in BD mice. The concentrations of Met metabolites, such as $S$-adenosylmethionine and $S$-adenosylhomocysteine were not significantly different between the two groups. The concentrations of glutathione and its reaction intermediates $\gamma$-Glu-Cys tendency to be lower in BD mice. The present study revealed that biotin deficiency induces an abnormal amino acids composition, especially among sulfur-containing amino acids and provide important information on the effect of biotin as a pharmacological agent.
\end{abstract}

Key Words biotin, metabolome analysis, sulfur-containing amino acids

Biotin is a water-soluble B-complex vitamin. The classical role of biotin is as a co-factor of 5 indispensable carboxylases: pyruvate carboxylase (PC), 2 isoforms of acetyl-CoA carboxylase (ACC1 and ACC2), propionylCoA carboxylase (PCC), and methylcrotonyl-CoA carboxylase (MCC) (1). These biotin-dependent carboxylases are involved in essential metabolic pathways, such as gluconeogenesis (PC), fatty acid synthesis (ACC1), fatty acid oxidation (ACC2), degradation of branchedchain amino acids and odd-chain fatty acids (PCC), and leucine catabolism (MCC). Holocarboxylase synthetase (HLCS) is a biotin ligase that catalyzes the biotinylation of the $\varepsilon$-amino group of a specific lysine residue in biotin-dependent carboxylases. Biotinidase (BTD) is an amidohydrolase that releases biotin from biotinylated peptides or biocytin (biotinyl- $\varepsilon-\mathrm{N}$-lysine) derived from the degradation of biotinylated carboxylases. Thus, cells can reuse the released-biotin to generate new holocarboxylases.

Because various foods contain biotin and mammals possess a biotin recycling system, severe dietary biotin deficiency is a rare with a normal diet. On the other hand, marginal biotin deficiency has been reported during pregnancy (2), and biotin deficiency in pregnant mouse dams affects embryonic development (3). Skin manifestations, such as alopecia and scaly and erythematous dermatitis, are induced by moderately severe biotin deficiency (4). We reported previously using a mouse model that biotin deficiency aggravated nickel allergy and biotin supplementation improved the allergic inflammation (5). Genetic defects of HLCS or BTD causes multiple carboxylase deficiency (MCD), a metabolic disorder resulting from reduced activities of the biotin-dependent carboxylases (1). Abnormal metabolites, such as 3-hydroxyisovaleric acid and 3-methylcrotonyl glycine resulting from reduced activity of MCC, have been reported as useful indicators for biotin deficiency (6). Neurological deficits are also induced by defects in BTD but not in HLCS (7). However, the precise mechanisms by which biotin deficiency induces various pathological conditions currently remains unclear.

Metabolomic analysis is a powerful method to elucidate metabolic disorders caused by nutrient deficiencies $(8)$. However, there are few reports on metabolomic analyses of biotin deficiency and/or MCD. In this study, we analyzed the metabolomic profiles of liver extracts taken from dietary biotin deficient mice. We showed that concentrations of sulfur-containing amino acids decreased significantly in liver extracts of biotin deficient mice. 


\section{Materials and Methods}

Female BALB/cA mice ( 4 wk old) were purchased from CLEA Japan, Inc. (Tokyo, Japan). Mice were divided into biotin-sufficient (BS) and biotin-deficient (BD) groups of 5 mice of each. These mice were fed for $8 \mathrm{wk}$ with a basal diet (AIN-76; $0.8 \mathrm{mg} / \mathrm{kg}$ of $d$-biotin) or a biotin-deficient (biotin-free) diet (Funabashi Farm Co., Funabashi, Japan), respectively (9). Mice were housed under controlled temperature $\left(23-25^{\circ} \mathrm{C}\right)$, a $12-\mathrm{h}$ dark/12-h light cycle (lights on from 7:00 to 19:00) with free access to water and chow diet until just before sampling. No clinical symptoms were detected in BD mice, and no significant differences of body weights were detected between BS and BD mice (9). All animal procedures were approved by the Institutional Animal Care and Use Committee of Tohoku University (approval number: 2012DnA-044 and 2013DnA-016) and performed in accordance with their guidelines.

Mice were killed between 13:00 and 15:00 by anesthetization with sevoflurane, and liver tissues were taken without prior food deprivation. Liver tissues were immediately frozen on dry-ice, and stored at $-80^{\circ} \mathrm{C}$ until further analysis. Liver tissue $(50 \mathrm{mg}$ ) was homogenized (5,000 rpm, $4^{\circ} \mathrm{C}, 30 \mathrm{~s}, 2$ times) using Precellys- 24 (Bertin Technologies, Washington D.C., USA) with zirconia beads and methanol containing $50 \mu \mathrm{mol} / \mathrm{L}$ methionine sulfone and D-camphor-10-sulfonic acid as internal controls. After the addition of $500 \mu \mathrm{L}$ of chloroform and $200 \mu \mathrm{L}$ of Milli- $Q$ water, the mixture was vortexed well and centrifuged with $2,300 \times g$ for $5 \mathrm{~min}$. The aqueous phase $(400 \mu \mathrm{L})$ was deproteinized by ultrafiltration ( $5 \mathrm{kDa}$ cutoff) and concentrated using a vacuum concentrator. The resultant samples were dissolved in $25 \mu \mathrm{L}$ of Milli-Q water. Water-soluble, low molecular weight metabolites were analyzed by capillary electrophoresis time-of-flight mass spectrometry (CE-TOFMS) (10).

Statistical analysis was performed with a Welch's two-tailed $t$-test and $p<0.05$ was considered significant (GraphPad Prism version 6.04, GraphPad Software, San Diego, CA, USA).

\section{Results and Discussions}

The concentrations of 10 of 126 metabolites (7.9\%) were significantly $(p<0.05)$ lower in liver extracts from $\mathrm{BD}$ mice than those from BS mice (Table 1). These comprised the concentrations of 5 essential amino acids, including Met (1.7-fold), Val (1.3-fold), Thr (1.3-fold), Ile (1.3-fold), and Leu (1.3-fold), and 2 conditionally essential amino acids, including Cys (2.0-fold) and Tyr (1.3-fold) were significantly lower in BD mice than BS mice. Among these, 2 sulfur containing amino acids, Cys and Met, were more than 1.5-fold lower in BD mice than in BS mice. MCC and PCC are involved with the catabolism of Leu and branched-chain amino acids (Ile, Thr and Val), respectively (1). Propionyl-CoA, a substrate for PCC, is a down-stream of a Met/Cys metabolism. MCC and PCC are sensitively influenced by biotin deficiency (11), suggesting that lower activity of each carboxylase suppressed the incorporation of the essen-

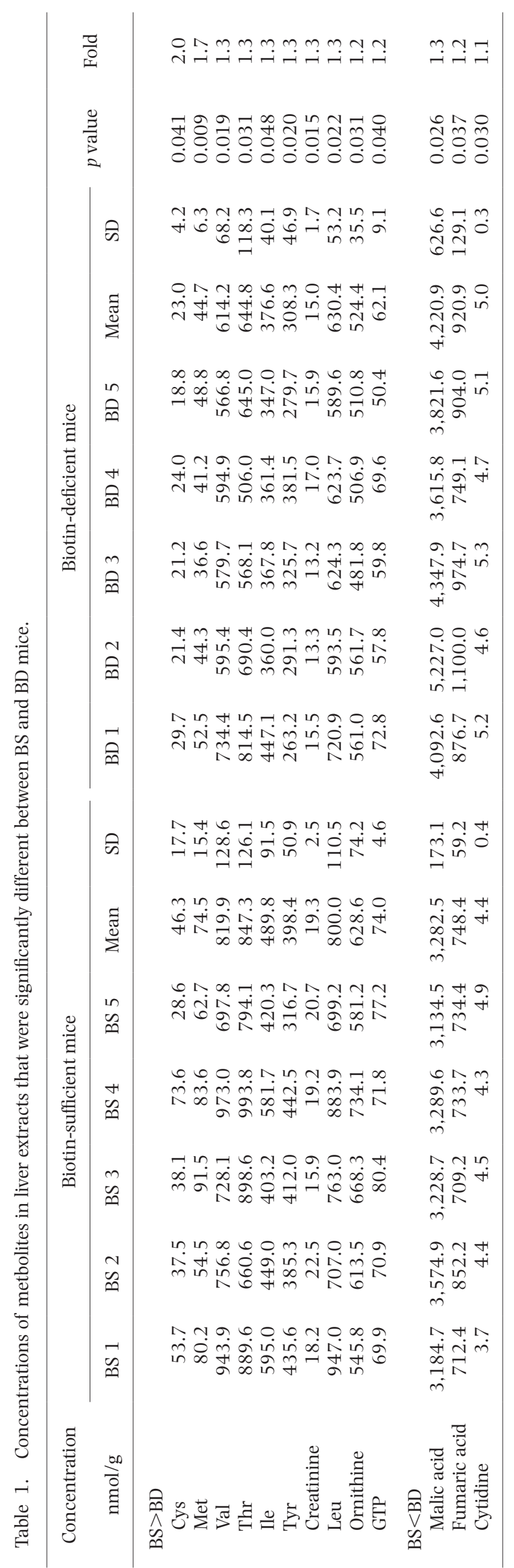


tial amino acids as a negative feedback.

Because Cys is synthesized from Met metabolism (Fig. 1A), Met metabolites in liver extracts were also analyzed. The concentrations of $S$-adenosylmethionine

(A)

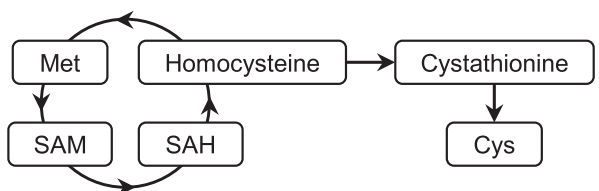

(B)

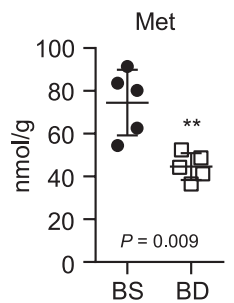

Homocysteine

ND
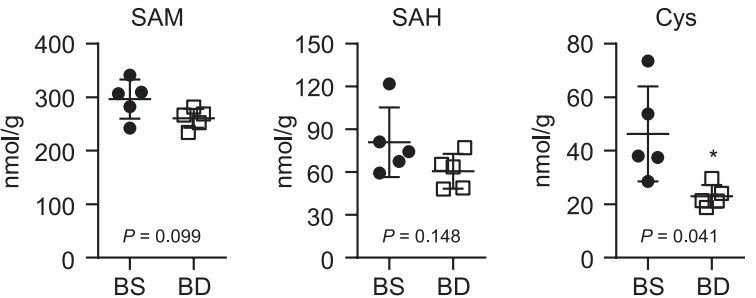

Fig. 1. The concentrations of Met metabolites in liver extracts prepared from BS and BD mice. (A) Schematic illustration of Met metabolism. (B) Concentrations of Met metabolites in liver extracts were measured using CE-TOFMS. Each symbol represents an individual mouse. Mean \pm SD is indicated. ${ }^{*} p<0.05,{ }^{* *} p<0.01$, significantly different from BS mice. ND, not detected.
(SAM) (1.1-fold, $p=0.099)$ and $S$-adenosylhomocysteine $(\mathrm{SAH})$ (1.3-fold, $p=0.148)$ were not significantly different between BS and BD mice (Fig. 1B). The concentration of cystathionine was 1.8-fold lower in the liver extracts from BD mice than those from BS mice, but the difference was not significant $(p=0.132)$. In agreement with published study which used same protocol (12), homocysteine was not detected in either group. Because homocysteine itself was detected by CE-TOFMS (10), extraction procedure is probably affected the detection of homocysteine. Although the concentrations of Met metabolites were not significantly lower in BD mice, it is suggested that a significantly lower concentration of Met affects the concentration of Cys. Moreover, Cys is finally catabolized to pyruvate (13). Decreased activity of PC in BD mice induces an accumulation of pyruvate, which may be a negative feedback for Cys biosynthesis and/or incorporation.

Antioxidant glutathione (GSH) is a major antioxidant in cells and composed of three amino acids, Glu, Cys and Gly (14). The first step of GSH biosynthesis is the $\gamma$-glutamyl bond between Glu and Cys which makes $\gamma$-Glu-Cys. Then, Gly is added to the C-terminus of $\gamma$-Glu-Cys. In the antioxidant process, GSH is converted to oxidized glutathione, glutathione disulfide (GSSG) (Fig. 2A). The concentrations of GSH (1.2-fold, $p=0.077)$ and $\gamma$-Glu-Cys (1.7-fold, $p=0.055)$ tended to be lower in liver extracts from BD mice than from BS mice (Fig. 2B). On the other hand, the concentrations of GSSG (1.0-fold, $p=0.374$ ) and GSH/GSSG ratio (1.1fold, $p=0.195$ ) were not significantly different between the groups. These results are consistent with previous studies that indicated Cys is a rate limiting amino acid for GSH biosynthesis (14), and Cys supplementation

(A)

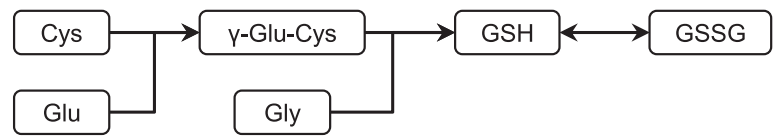

(B)
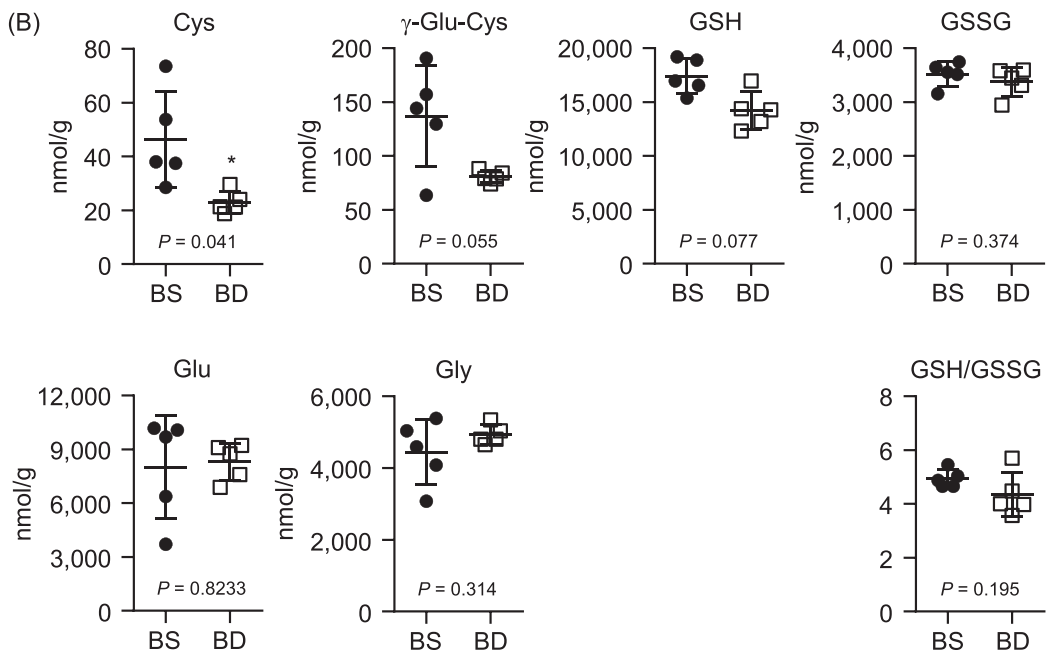

Fig. 2. The concentrations of GSH and its related metabolites in liver extracts prepared from BS and BD mice. (A) Schematic illustration of GSH biosynthesis pathway. (B) Concentrations of GSH and its related metabolites in liver extracts were measured using CE-TOFMS. Each symbol represents an individual mouse. Mean \pm SD is indicated. * $p<0.05$, significantly different from BS mice. 
upregulated GSH expression in the livers of mice fed on a low protein diet (15). Moreover, Richie et al. reported that Met restriction induced decreases of Cys and GSH in liver (16), consistent with the present result that the concentration of Met was also significantly lower in liver extracts from BD mice than from BS mice.

Concentrations of creatinine (1.3-fold), ornithine (1.2-fold), and GTP (1.2-fold) were also significantly lower in the liver extracts from BD mice than those from BS mice, but the differences were less than 1.5fold (Table 1). Concentrations of 3 metabolites (3/126, $2.4 \%$ ), including malic acid (1.3-fold), fumaric acid (1.2fold) and cytidine (1.1-fold), were significantly ( $p<0.05)$ higher in liver extracts from BD mice than those from BS mice, but the differences were less than 1.5-fold.

In this study, we analyzed the metabolomic profiles of liver extracts prepared from BS and BD mice and found that the concentrations of sulfur-containing amino acids, Cys and Met, were significantly lower in liver extracts from BD mice than from BS mice. Our protocol induced no significant differences of body weight between BS and BD mice (9), suggesting that decrease of food intake did not cause the significant decreases in amino acids. To the best of our knowledge, this is the first study reporting metabolomic analysis of BD animals. Biotin shows pharmacological functions against various diseases, such as diabetes (17), antihypertension (18), and metabolic syndrome (19). However, the mechanisms of how biotin exerts these pharmacological function remain unclear. The present study revealed that biotin deficiency induces abnormal amino acids composition, especially among sulfur containing amino acids. Our results provides important information on the use of biotin as a preventive and/or therapeutic agent against various diseases.

\section{Acknowledgments}

This work was supported in part by the Japan Society for the promotion of Science KAKENHI Grant Numbers, 23792140 (T.K.) and 25462913 (T.K.). We thank the Biomedical Research Core of Tohoku University Graduate School of Medicine for the metabolome analysis.

\section{Disclosure of state of COI}

The authors declare no commercial or financial conflict of interest.

\section{REFERENCES}

1) Zempleni J, Wijeratne SSK, Kuroishi T. 2012. Biotin. In: Present Knowledge in Nutrition, Tenth ed (Erdman JW Jr, Macdonald IA, Zeisel SH, eds), p 359-374. John Wiley \& Sons, Iowa.

2) Mock DM. 2009. Marginal biotin deficiency is common in normal human pregnancy and is highly teratogenic in mice. J Nutr 139: 154-157.

3) Watanabe T, Nagai Y, Taniguchi A, Ebara S, Kimura S, Fukui T. 2009. Effects of biotin deficiency on embryonic development in mice. Nutrition 25: 78-84.
4) Mock DM. 1991. Skin manifestations of biotin deficiency. Semin Dermatol 10: 296-302.

5) Kuroishi T, Kinbara M, Sato N, Tanaka Y, Nagai Y, Iwakura Y, Endo Y, Sugawara S. 2009. Biotin status affects nickel allergy via regulation of interleukin-1beta production in mice. J Nutr 139: 1031-1036.

6) Mock DM, Henrich CL, Carnell N, Mock NI. 2002. Indicators of marginal biotin deficiency and repletion in humans: validation of 3-hydroxyisovaleric acid excretion and a leucine challenge. Am J Clin Nutr 76: 1061-1068.

7) Wolf B. 2011. The neurology of biotinidase deficiency. Mol Genet Metab 104: 27-34.

8) Astarita G, Langridge L. 2013. An emerging role for metabolomics in nutrition science. J Nutrigenet Nutrigenomics 6: 181-200.

9) Kuroishi T, Endo Y, Muramoto K, Sugawara S. 2008. Biotin deficiency up-regulates TNF-alpha production in murine macrophages. J Leukoc Biol 83: 912-920.

10) Ohashi Y, Hirayama A, Ishikawa T, Nakamura S, Shimizu K, Ueno Y, Tomita M, Soga T. 2008. Depiction of metabolome changes in histidine-starved Escherichia coli by CE-TOFMS. Mol Biosyst 4: 135-147.

11) Eng WK, Giraud D, Schlegel VL, Wang D, Lee BH, Zempleni J. 2013. Identification and assessment of markers of biotin status in healthy adults. Br J Nutr 110: 321-329.

12) Soga $T$, Baran $R$, Suematsu $M$, Ueno $Y$, Ikeda $S$, Sakurakawa T, Kakazu Y, Ishikawa T, Robert M, Nishioka T, Tomita M. 2006. Differential metabolomics reveals ophthalmic acid as an oxidative stress biomarker indicating hepatic glutathione consumption. J Biol Chem 281: 16768-16776.

13) Bender DA. 2012. The metabolism of "surplus" amino acids. Br J Nutr 108: S113-121.

14) Wu G, Fang YZ, Yang S, Lupton JR, Turner ND. 2004. Glutathione metabolism and its implications for health. J Nutr 134: 489-492.

15) Hunter EA, Grimble RF. 1994. Cysteine and methionine supplementation modulate the effect of tumor necrosis factor alpha on protein synthesis, glutathione and zinc concentration of liver and lung in rats fed a low protein diet. J Nutr 124: 2319-2328.

16) Richie JP Jr, Komninou D, Leutzinger Y, Kleinman W, Orentreich N, Malloy V, Zimmerman JA. 2004. Tissue glutathione and cysteine levels in methionine-restricted rats. Nutrition 20: 800-805.

17) Lazo de la Vega-Monroy ML, Larrieta E, German MS, Baez-Saldana A, Fernandez-Mejia C. 2013. Effects of biotin supplementation in the diet on insulin secretion, islet gene expression, glucose homeostasis and beta-cell proportion. J Nutr Biochem 24: 169-177.

18) Watanabe-Kamiyama M, Kamiyama S, Horiuchi K, Ohinata K, Shirakawa H, Furukawa Y, Komai M. 2008. Antihypertensive effect of biotin in stroke-prone spontaneously hypertensive rats. Br J Nutr 99: 756-763.

19) Aguilera-Mendez A, Hernandez-Equihua MG, RuedaRocha AC, Guajardo-Lopez C, Nieto-Aguilar R, SerratoOchoa D, Ruiz Herrera LF, Guzman-Nateras JA. 2018. Protective effect of supplementation with biotin against high-fructose-induced metabolic syndrome in rats. Nutr Res 57: 86-96. 